\title{
Malignancy in a Family with Carney's Complex
}

\author{
Sean Roy Beh, ${ }^{1 *}$ Khalid Abdul Kadir ${ }^{2}$ \\ ${ }^{1}$ Monash University Malaysia, Malaysia \\ ${ }^{2}$ Endocrine, Internal Medicine, Monash University Malaysia, Malaysia
}

\begin{abstract}
Carney Complex is a genetic disease characterized by skin lentigenes and freckling and the growth of benign tumors in endocrine glands as well as cardiac myxoma. Malignant tumors in Carney's Complex are extremely rare with few other case reports. We report the rare occurrence of ovarian malignancy and thyroid carcinoma separately in 2 out of 3 family members diagnosed with Carney Complex.
\end{abstract}

Keywords: Carney Complex, Cardiac myoxomata, Eendocrine tumors, Chemotherapy, Malignant tumor

Learning Points

a) The importance of thoroughly screening all lesions in Carney's Complex rather than dismissing them as benign, as there is still a possibility for the lesion to be malignant.

b) Malignant tumors in Carney's complex can arise from any organ, which in the index case presented with multiple tumors over many years culminating in an ovarian malignant tumor, whereas the sister developed a thyroid malignancy.

c) Acromegaly occurring in multiple members of a family should raise the suspicion of Carney's complex to the treating clinician.

d) All other family members of a patient presenting with Carney's Complex should be screened as they are at risk of also developing Carney's complex.

\section{Background}

Carney complex (CNC), first discovered by J. Aidan Carney, is a neoplastic endocrine disorder characterized by tumor growth that affects the skin, adrenal glands, pituitary gland, thyroid gland, heart, reproductive organs, bones, and nerves. It is a rare disease with only around 750 cases reported by including patients from all continents. With genotyping of a large cohort (350 patients), it was shown that CNC affects females more than males, where in $70 \%$ of the cases, it is inherited through an autosomal dominant pattern with a mutation in the PRKAR1A gene on 17q24.2-24.3 causing loss of function. Through genetic linkage study, another gene suspected to contribute to CNC is located on chromosome 2p16. However, most of the tumor manifestations mentioned is likely to be benign in nature. ${ }^{1-4}$

\section{Diagnostic Criteria for Carney's Complex}

To diagnose Carney's Complex, at least two manifestations from Table 1, or one criterion with any one of the following supplemental criteria: affected first-degree relative or inactivating mutation of the PRKAR1A gene. ${ }^{1}$

\section{Methods}

A Malaysian family with Carney's Complex (CNC) was identified and clinical evaluation was done along with laboratory tests. The family members are brought to the clinic for their routine check-up and monitoring of their past complications of CNC. Their clinical presentations were compared with current literature to identify their risks of developing further complications of CNC and then the

\begin{tabular}{|l|l|}
\hline Quick Response Code: & *Corresponding author: Sean Roy Beh, Monash University Malaysia, Jalan Lagoon Selatan, \\
Bandar Sunway, 47500 Subang Jaya, Selangor, Malaysia \\
Received: 18 February, 2021 & Published: 17 March, 2021 \\
\hline
\end{tabular}


risks were weighed against the need for additional screening between the different family members. For this discussion, we will be focusing on the oldest sibling within the family who developed an ovarian malignancy.

Table 1: Shows major criteria for the diagnosis of Carney's Complex'.

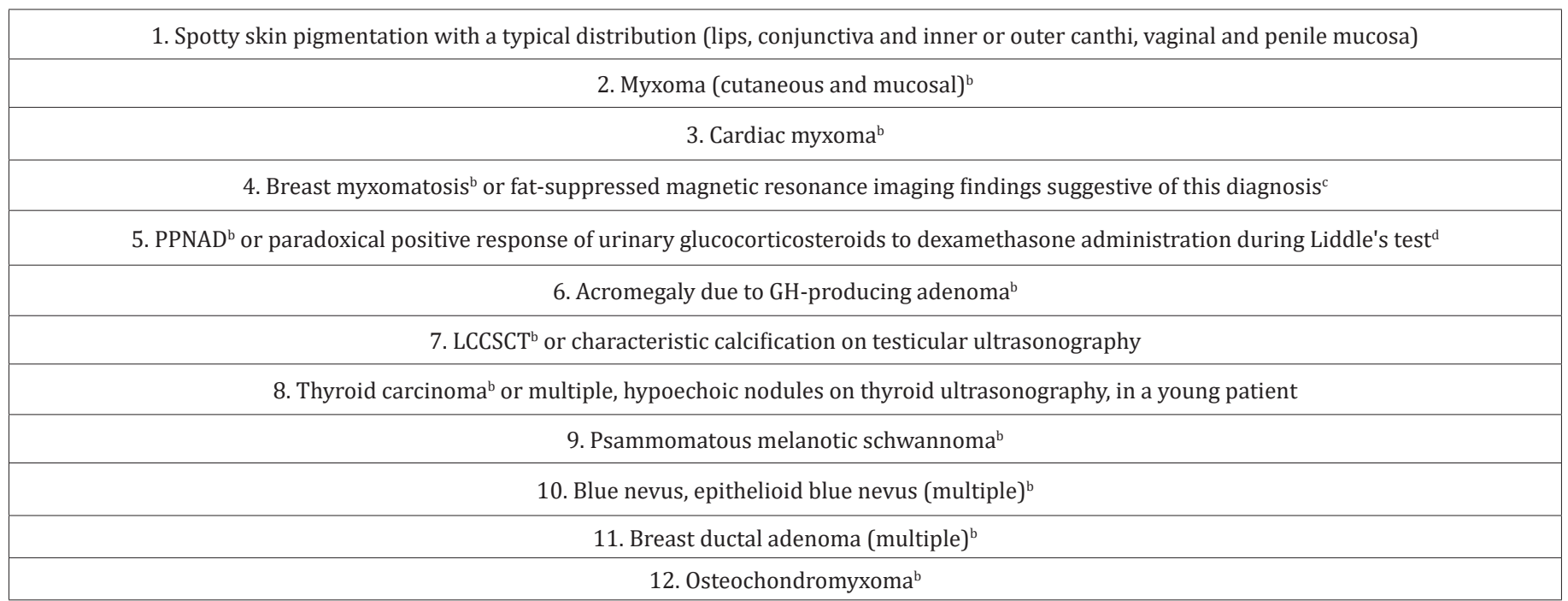

\section{Case Presentation}

A 60-year-old Malaysian lady with intense facial freckling was diagnosed to have acromegaly when she presented with pituitary apoplexy 22 years ago with bitemporal loss of vision. This was treated surgically, but complicated by CSF rhinorrhea and local infection. She was noted then to have undiagnosed gross features of acromegaly and to have facial freckles and lentigenes consistent with Carney's Complex.She became hypopitutary and 14 years ago following multiple small strokes she was found to have myxomata in both atria and left ventricles which were respected. Later she had an obstructive benign thyroid goiter surgically removed. Three years ago she developed lower abdominal pain and post-menopausal bleeding. Investigations showed a cystic lesion in the left ovary. Total abdominal hysterectomy bilateral salphingo oophorectomy (TAHBSO) was performed. The histology of the tumor was a metastatic mucinous adenocarcinoma (Left to Right ovary). No further metastases to other sites were detected. She was given a course of chemotherapy with no evidence of recurrence for the last 4 years of follow-up.

Her younger sister who also has freckles and lentigenes, developed Cushing 's disease due to bilateral pigmented nodular adrenal hyperplasia and underwent bilateral total adrenalectomy. 3 years ago she developed a papillary thyroid carcinoma, treated surgically with one episode of recurrence and ablative radiotherapy. She remains free of further recurrence.

\section{Another sister is free of disease}

The only brother, who is also the youngest sibling, has multiple cardiac myoxomata requiring surgical resection and also active acromegaly but no other tumors. Their mother and aunt died early of unknown causes, but the father lived to old age and died of lung cancer associated with smoking. The family members on the father's side are all free of the disease. The patient is single. The children of her siblings are free of disease at the moment.

\section{Treatment \& Investigation}

The ovaries and uterus were removed surgically. The left ovarian tumor was sent for histopathology. Microscopy of sections of the left ovarian cyst show invasion by complex malignant glands lined by stratified non-mucinous containing epithelium. The nuclei are round and hyper chromatic and cytoplasm is ample. Well differentiated tubular glands and papillary formations are also present. Squamous morules are seen in foci. Some areas show small rosette like glands. These complex glands are seen to invade the stroma surrounded by a marked desmoplastic reaction. No capsular invasion is seen. No tumor cells are seen on ovarian surface. The left fallopian tube shows absence of tumor cells.

The right ovary shows foci of tumour metastases in forms of well-formed malignant glands lined by atypical epithelium, surrounded by desmoplastic stroma. Other sections of the cord, uterus, and cervix were unaffected.

The diagnosis of left ovarian endometrioid adenocarcinoma with right ovarian metastatic endometrioid adenocarcinoma was given. TNM Stage T1bNxMx. FIGO stage 1B.She was given XC course of chemotherapy and local Deep X-ray therapy to the tumorsite. There were however no further incidence of metastases.

Images of the respected ovaries are attached in the Appendix section in the final page.

\section{Results}

Within the Malaysian family, CNC appears to affect 3 of the 4 siblings, which is coherent to an autosomal dominant pattern. ${ }^{1-4}$ The manifestations were typical and progressive, as the siblings developed freckles and tumors at different times in their lives ranging from pituitary tumours causing acromegaly, atrial myxomatas which required open heart surgery, Cushing's syndrome and thyroid nodules. ${ }^{1-4}$ However, two siblings developed ovarian cancer and thyroid cancer respectively, which is not only a rare manifes- 
tation of CNC but carries a very high mortality rate..$^{5-7}$ We also want to emphasize that freckles are uncommon in an Asian population, more so in an entire Malaysian family presenting with freckles. Therefore, a high degree of suspicion should be there if a multitude of tumors were present in a patient with freckling at a clinical site.

\section{Outcome and Follow-Up}

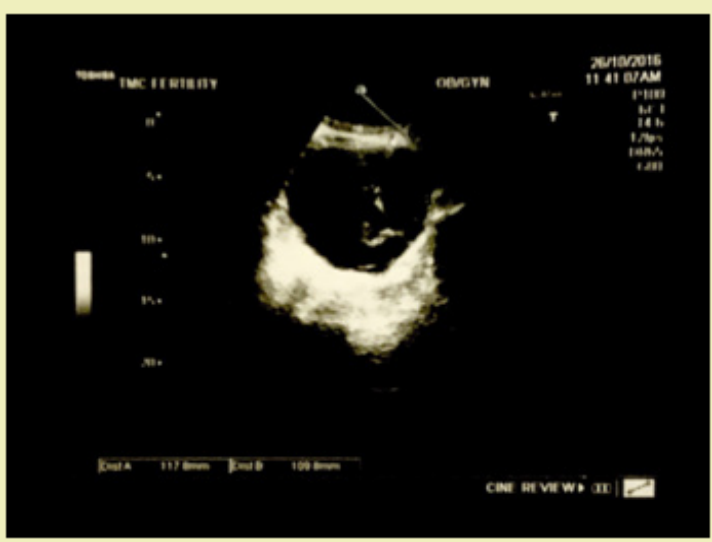

Figure 1: Appendix.

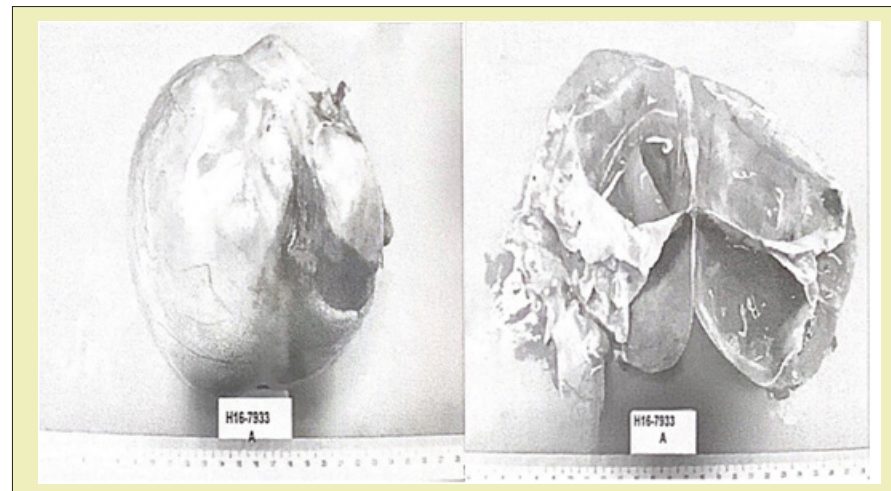

Figure 2: Ultrasound Image of patient from Case A: Left Ovary.

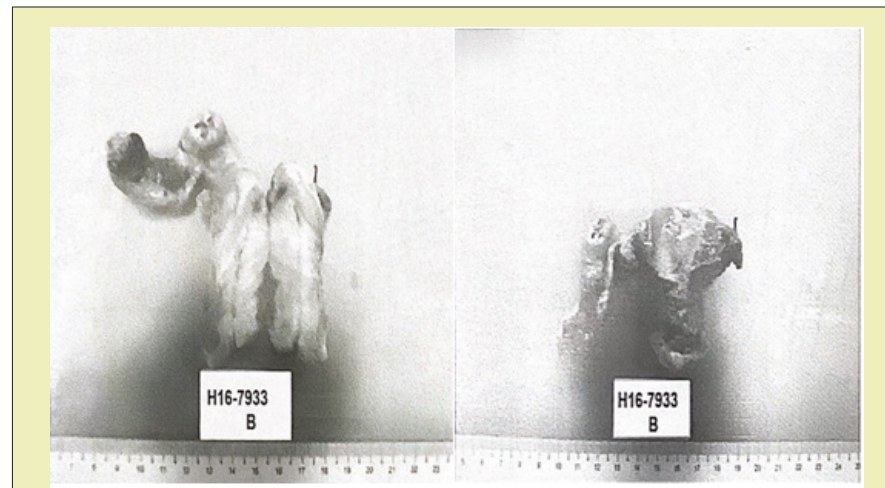

Figure 3: Tumour specimen of patient from Case A: Left ovary; Tumour specimen of patient from Case A: Uterus and right tube and ovary.

Throughout 4 years of follow-up, the patient and other family members came in for their routine scheduled annual clinical reviews regarding the hormonal replacements for removal of endocrine organs as well as progression or complications arising from the surgical treatments. If there were any recurrence of tumors that lead to obstructive symptoms or presence of any suspicious growths, further surgical treatments would be offered. For the entire follow-up, there seems to be no recurrence or development of malignancies or problematic tumors in any endocrine organs for the patient or any of the other family members (Figures 1-3).

\section{Discussion}

Carney Complex is an endocrine disease characterized by growth of benign tumors, and development of malignant tumors' are extremely rare. We report on a family where 2 out of 3 siblings diagnosed with Carney's complex developed a form of malignant tumor, a left ovarian endometrioid adenocarcinoma with right ovarian metastatic endometrioid adenocarcinoma, and a papillary thyroid carcinoma separately.

In one other case report that described an ovarian malignancy in Carney's Complex, FISH analysis shown that there is an amplification of homogenously-staining region on $2 \mathrm{p} 16$ which is a gene loci associated with Carney's Complex.5,6 Though genetic screening would be useful in predicting the development of ovarian malignancies, more conventional methods of screening in patients with Carney's Complex such as ultrasound should be used instead for detection of tumors initially.

As for thyroid carcinomas in Carney's Complex, the more common thyroid malignancy is follicular carcinoma which carries an extremely poor prognosis and is often non-responsive to radioiodine or lenvatinib treatment.7The patient's sister however presented with a papillary carcinoma which carries a much better prognosis and responsiveness to radioiodine treatment which she was given.

A genetic analysis of these 2 malignant tumors' mentioned above would give us a clearer picture whether there is a genetic link between these 2 malignancies developing in different siblings diagnosed with Carney's complex, and whether they have amplifications in similar gene foci, putting one sibling at risk of developing similar malignant cancers as the other sibling, and vice versa.

This case report emphasizes the need to thoroughly screen endocrine tumors arising in Carney's Complex as, although rare, they may be malignant in nature.

\section{Acknowledgments}

None.

\section{Funding}

None.

\section{Conflicts of interest}

Author declares that there is no conflict of interest.

\section{References}

1. Correa R, Salpea P, Stratakis CA. Carney complex: an update. Eur J Endocrinol. 2015;173(4):M85-M97.

2. Malchoff CD. Carney Complex-Clarity and Complexity. J Clin Endocrinol Metab. 2000;85(11):4010-4012.

3. Horvath A, Stratakis CA. Clinical and molecular genetics of acromegaly: MEN1, Carney complex, McCune-Albright syndrome, familial 
acromegaly and genetic defects in sporadic tumors. Rev Endocr Metab Disord. 2008;9(1):1-11.

4. Stratakis CA, Raygada M. Carney Complex. In: Adam MP, Ardinger $\mathrm{HH}$, Pagon RA, et al., Editors. GeneReviews ${ }^{\circledR}$ [Internet]. Seattle (WA): University of Washington, Seattle; 2003:1993-2020.

5. Stratakis CA, Papageorgiou T, Premkumar A, et al. Ovarian Lesions in Carney Complex: Clinical Genetics and Possible Predisposition to Malignancy. J Clin Endocrinol Metab. 2000;85(11):4359-4366.
6. Papageorgiou T, Stratakis CA. Ovarian tumors associated with multiple endocrine neoplasias and related syndromes (Carney complex, PeutzJeghers syndrome, von Hippel-Lindau disease, Cowden's disease). Int J Gynecol Cancer. 2002;12(4):337-347.

7. Carney JA, Lyssikatos C, Seethala RR, et al. The Spectrum of Thyroid Gland Pathology in Carney Complex: The Importance of Follicular Carcinoma. Am J Surg Pathol. 2018;42(5):587-594. 